\title{
Desempenho das Simulações do Projeto Cordex Quanto à Representação dos Padrões de Variação da Precipitação no Século XX sobre o Município de Fortaleza, Ceará
}

\author{
Antonio Elder Ferreira da Silva ${ }^{1}$ (D), Davi Teixeira Gomes ${ }^{1}$, Cleiton da Silva Silveira ${ }^{2}$, \\ Meiry Sayuri Sakamoto ${ }^{1}$ \\ ${ }^{1}$ Centro de Ciências e Tecnologia, Universidade Estadual do Ceará, Fortaleza, CE, Brasil. \\ ${ }^{2}$ Departamento de Engenharia Hidráulica e Ambiental, Centro de Tecnologia, \\ Universidade Federal do Ceará, Fortaleza, CE, Brasil.
}

Recebido em: 2 de Abril de 2020 - Aceito em: 28 de Maio de 2020

\begin{abstract}
Resumo
O método de avaliação aplicado foi o proposto por Silveira et al. (2019). A pesquisa buscou avaliar simulações dos modelos do Projeto Cordex para o domínio da América do Sul que fornecessem dados de precipitação em frequência mensal da família do experimento Historical para município de Fortaleza, Ceará. Foram encontrados quatro RCMs aninhados com dez GCMs totalizando dezesseis combinações denominadas aqui simplesmente por "modelos". Para realizar a comparação foram utilizados dados observados da Estação Pici (338001) obtidos no Portal HidroWeb durante período de 1970 a 2005. O modelo regional ETA apresentou o melhor desempenho na representatividade do clima presente. A condução pelo modelo global CanESM2, segundo o índice AVALs, se apresentou como melhor simulação para o período avaliado com um CORREL de 0,96 e RMSE_PC de 3,01\%. Ficou evidenciado a relação direta da ordem de classificação com espaçamento das grades, ou seja, quanto maior a resolução do modelo mais bem posicionado na classificação. Após a avaliação desses modelos, pesquisadores interessados em aplicar dados de modelagem em Fortaleza terão arcabouço científico para adotar modelos adequados em suas pesquisas. Em tese, os modelos que conseguem representar de forma adequada o clima presente, possivelmente também conseguirão representar o clima futuro.
\end{abstract}

Palavras-chave: Cordex, CMIP5, modelo climático, mudanças climáticas.

\section{Performance of the Cordex Project Simulations with Regard to the Representation of the Patterns of Variation of the Precipitation in the XX Century on the Municipality of Fortaleza, Ceará}

\begin{abstract}
The evaluation method applied was the one proposed by Silveira et al. (2019). The research sought to evaluate simulations of the Cordex Project models for the South America domain that provide monthly frequency rainfall data from the Historical experiment family for the city of Fortaleza, Ceará. Four RCMs were found nested with ten GCMs totaling sixteen combinations named here simply by "models". To perform the comparison, data from the Pici Station (338001) obtained from the HidroWeb Portal during the period from 1970 to 2005 were used. The regional ETA model presented the best performance in the representativeness of the present climate. Driving by the global CanESM2 model, according to the AVALs index, was the best simulation for the period evaluated with a CORREL of 0.96 and RMSE_PC of 3.01\%. The direct relationship between the classification order and grid spacing was evident, that is, the higher the resolution of the model, the better positioned in the classification. After evaluating these models, researchers interested in applying modeling data in Fortaleza will have a scientific framework to adopt appropriate models in their research. In theory, models that can adequately represent the present climate, possibly also will be able to represent the future climate.
\end{abstract}

Keywords: Cordex, CMIP5, model climate, climate change.

Autor de correspondência: Antonio Elder Ferreira da Silva, elderferreira2007@gmail.com. 


\section{Introdução}

Atualmente, tem-se discutido arduamente sobre a motivação das alterações climáticas. A alteração do clima, segundo a United Nations Framework Convention on Climate Change (UNFCC) pode ocorrer de duas maneiras distintas: pela alteração devida à interação humana com o meio ambiente, de maneira que altera a composição do solo e da atmosfera global; e pela forma natural, ou seja, devido a características da evolução da terra ao longo do tempo (Costa, 2016).

Nas últimas décadas, a comunidade científica tem registrado aumentos significativos na concentração dos gases de efeito estufa (GEE), em especial, do dióxido de carbono $\left(\mathrm{CO}_{2}\right)$. Segundo especialistas, esses aumentos foram provocados pelo desenvolvimento de atividades antrópicas desde a revolução industrial até os dias atuais. Os gases responsáveis por esse efeito estufa absorvem parte das radiações infravermelhas que a terra irradia para o espaço, provocando uma retenção de calor, o que acarretou um aquecimento de aproximadamente $0,5^{\circ} \mathrm{C}$ na temperatura média da superfície terrestre no último século (Rossi; Dumke; Kruger, 2009). Esse aquecimento global está sendo considerado como gatilho dessas alterações do clima.

Já é reconhecido que as cidades são as principais contribuintes para essas emissões. Os centros urbanos são responsáveis pelo consumo de $70 \%$ da energia disponível e por $40 \%$ das emissões de GEE (Rosenzweig et al., 2015). Hoje, mais da metade da população mundial (3,6 bilhões) vive em cidades. Em 2050, é esperado que a população urbana cresça de 5,6 para 7,1 bilhões, ou $64 \%$ para $69 \%$ da população mundial (PBMC, 2016).

Da mesma forma que as cidades se apresentam como principal vetor dessas emissões, também serão as principais prejudicadas com essa postura. Os principais problemas envolvendo mudanças climáticas e cidades são o aumento de temperatura, aumento no nível do mar, ilhas de calor, inundações, escassez de água e alimentos, acidificação dos oceanos e eventos extremos. Segundo PBMC (2016), a maioria das cidades brasileiras já tem problemas ambientais associados a padrões de desenvolvimento e transformação de áreas geográficas.

Por isso, é importante que o planejamento das cidades inclua o conhecimento das vulnerabilidades existentes e riscos associados à ocorrência de eventos climáticos extremos. $\mathrm{O}$ ideal é que as cidades sejam remodeladas e planejadas de acordo com as prioridades existentes, visando torná-las resilientes às mudanças climáticas e adeptas aos padrões de desenvolvimento sustentável.

Nessa perspectiva, a modelagem climática se apresenta como ferramenta para auxiliar o planejamento dos aglomerados urbanos. Os grandes centros de pesquisas climáticas simulam, através de modelos numéricos, os processos físicos na atmosfera, nos oceanos, na criosfera e na superfície terrestre. Atualmente, são as ferramentas disponíveis mais avançadas para simular a resposta do sistema climático às crescentes concentrações de gases do efeito estufa (IPCC, 2007). Esses modelos oferecem diversas variáveis de saída, em especial, dados de precipitação e temperatura. Os prognósticos das simulações podem ajudar a compreender os impactos e auxiliar na adoção de medidas que promovam a mitigação e adaptação frente as mudanças climáticas.

O Intergovernmental Panel on Climate Change (IPCC) é uma organização científico-política que tem como objetivo sintetizar e divulgar o conhecimento mais avançado sobre mudanças climáticas. O IPCC utiliza-se dos Global Climate Models (GCMs) como ferramentas para realizar um prognóstico do clima mundial. Os GCMs projetam para o futuro variáveis climáticas com base nas emissões dos gases de efeito estufa em conjunto com fatores como demografia, desenvolvimento socioeconômico e mudança na tecnologia (IPCC, 2001).

Segundo Ramos (2010), a academia científica já reconhece que os GCMs fornecem boas projeções em escala de tempo mensal e anual, especialmente para variáveis como a precipitação. Entretanto, esses dados se referem a contornos de clima global (baixa resolução) não considerando características de nível local como relevo, desmatamento, atividades agrícolas, uso indevido da terra, expansão da área urbana, entre outras. Para realizar essa adequação o presente estudo utilizou dados de Regional Climate Models (RCMs) integrados pelo Projeto Cordex (Coordinated Regional Climate Downscaling Experimente). Trata-se de uma iniciativa do WCRP (World Climate Research Programme) que fornece informação climática de alta resolução obtida por donwnscaling dinâmico ou estatístico de modelos globais (Benestad, 2016).

Tendo em vista que os impactos das mudanças climáticas deverão ocorrer em escala regional e que as cidades serão as mais afetadas, o presente estudo buscou avaliar a capacidade de alguns modelos regionais de clima representarem os padrões de variação da precipitação mensal no município de Fortaleza no Estado do Ceará, Brasil. A ideia é que após a avaliação desses modelos, pesquisadores interessados em aplicar dados de modelagem para o município estudado possuam arcabouço científico para adotar modelos adequados em suas pesquisas. Em tese, os modelos que conseguem representar de forma adequada o clima presente, possivelmente também conseguirão representar o clima futuro (Silveira et al., 2019). As simulações de precipitação mensal podem ser aplicadas na estimativa de impactos em diversas áreas do conhecimento, por exemplo, em estudos que envolvam aproveitamento de água da chuva, dimensionamento de dispositivos de drenagem urbana, associação entre enchentes urbanas e proliferação de determinadas doenças, dentre outras temáticas relacionáveis com as mudanças climáticas. 
Contudo, apesar de pautada em bases científicas, as incertezas envolvidas no processo de modelagem climática podem não representar de forma fidedigna o clima futuro. A sociedade em geral deve compreender que essas incertezas existem e que os resultados encontrados em pesquisas dessa natureza não podem ser tratados como verdade absoluta. Os resultados apontam apenas para uma possibilidade de ocorrência.

Esta pesquisa teve o objetivo avaliar o desempenho de modelos climáticos regionais quanto a representação da sazonalidade da precipitação média mensal do município de Fortaleza, Estado do Ceará, Brasil. Para alcance desse objetivo foi aplicado o método de avaliação proposto por Silveira et al. (2019) que consiste basicamente nas comparações de dados observados e simulados por modelos climáticos para o clima presente (Século XX).

\section{Materiais e Métodos}

A presente pesquisa foi realizada com dados do regime pluviométrico do município de Fortaleza, no estado do Ceará (Fig. 1). A capital cearense, que pertence à região Metropolitana de Fortaleza, desenvolveu-se às margens do riacho Pajeú, no Nordeste do Brasil, a 2.285 quilômetros de Brasília. Segundo o Instituto de Pesquisa e Estratégia Econômica do Ceará (IPECE), Fortaleza possui clima Tropical Quente Sub-úmido, com pluviosidade média de $1.338 \mathrm{~mm}$, apresenta temperatura média de $26{ }^{\circ} \mathrm{C}$ a $28{ }^{\circ} \mathrm{C}$ e indica período chuvoso de janeiro a maio (CEARÁ, 2018).

Na grande maioria dos trabalhos que avaliaram modelos, os dados de observação foram extraídos de modelos numéricos que tentam espacializar dados meteorológicos em grades (dados reconstruídos), como por exemplo, o CRU (Climate Research Unit). Para o presente estudo experimentou-se outra fonte de dado observado para comparar a destreza dos modelos no clima presente (Século $\mathrm{XX}$ ). Considerando que nesta pesquisa a escala geográfica envolve apenas a área de um município, foi utilizado dado obtido de estação meteorológica de superfície, ou seja, o dado bruto nas suas condições reais de ocorrência e não interpolados por estimativa numérica.

A aquisição da série de precipitação mensal se deu por meio do Portal HidroWeb (ANA, 2019). Foi coletado valores que compreendem o período de 1970 a 2005 da estação 338001 (Pici) de coordenadas geográficas latitude $-3.75^{\circ}$ e longitude $-38.58^{\circ}$. A escolha dessa estação se justifica pelo fato de ser a única dentro do território do munícipio que possui dados observados desse período sem falhas de preenchimento.

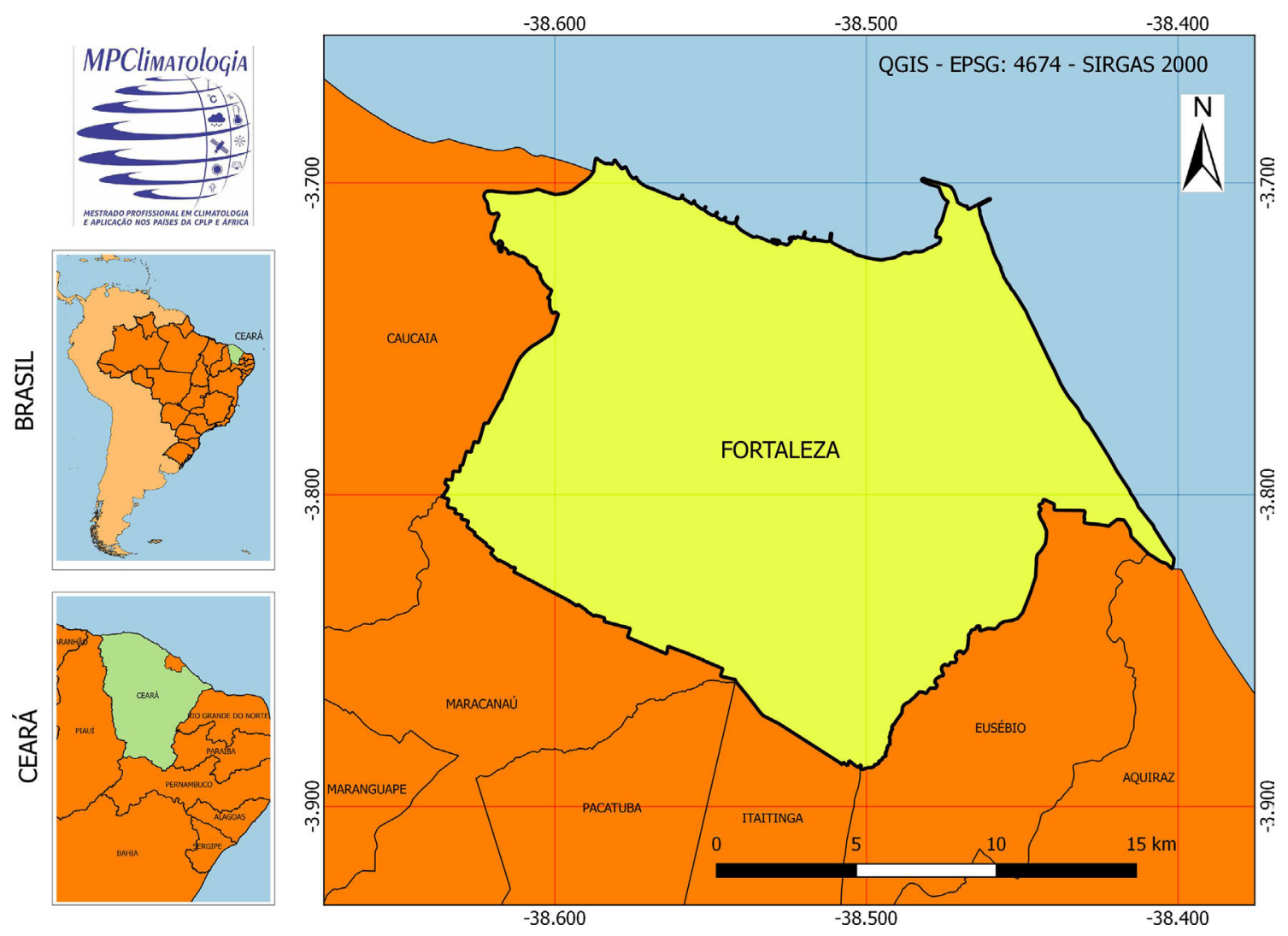

Figura 1 - Localização do município de Fortaleza. Está localizada no litoral Atlântico (Latitude: -3.72; Longitude: -38.54), com 34 km de praias, a uma altitude média de 21 metros, possui 313,8 $\mathrm{km}^{2}$ de área e 2.500.194 habitantes, sendo a capital de maior densidade demográfica do país, com 7.815,7 hab/ $\mathrm{km}^{2}$, é a cidade mais populosa do Ceará, a quinta maior capital do Brasil e a $91^{\mathrm{a}}$ mais populosa do mundo (Fortaleza, 2015). 
Definida a estação de observação e coletado seus dados, o passo seguinte foi a escolha dos modelos climáticos disponíveis segundo parâmetros de pesquisa como variável, frequência, família de experimento, domínio, dentre outros. Antes disso se faz necessária as seguintes explicações.

Os modelos globais (GCMs) podem fornecer projeções de como o clima da Terra pode mudar no futuro. Esses resultados são a principal motivação para a comunidade internacional tomar decisões sobre a mitigação das mudanças climáticas. No entanto, os impactos de um clima em mudança e as estratégias de adaptação necessárias para lidar com eles ocorrerão em escalas regionais. É aqui que os modelos regionais (RCMs) tem um papel importante a desempenhar ao fornecer projeções com muito mais detalhes e uma representação mais precisa dos eventos extremos localizados. Na tentativa de padronizar as simulações climáticas de diversos centros de pesquisa pelo mundo, foram criados projetos coordenados de comparação dos resultados dos modelos climáticos. Para os GCMs, tem-se o CMIP (Coupled Model Intercomparison Project), e para os RCMs, o Projeto Cordex (Benestad, 2016).

As condições de contorno dos RCMs são balizadas pelos GCMs integrantes do CMIP fase 5. Os períodos de tempo utilizados são divididos em Historical (século XX) e projeções (século XXI). Os cenários recomendados estão associados a cenários futuros (Representative Concentra-

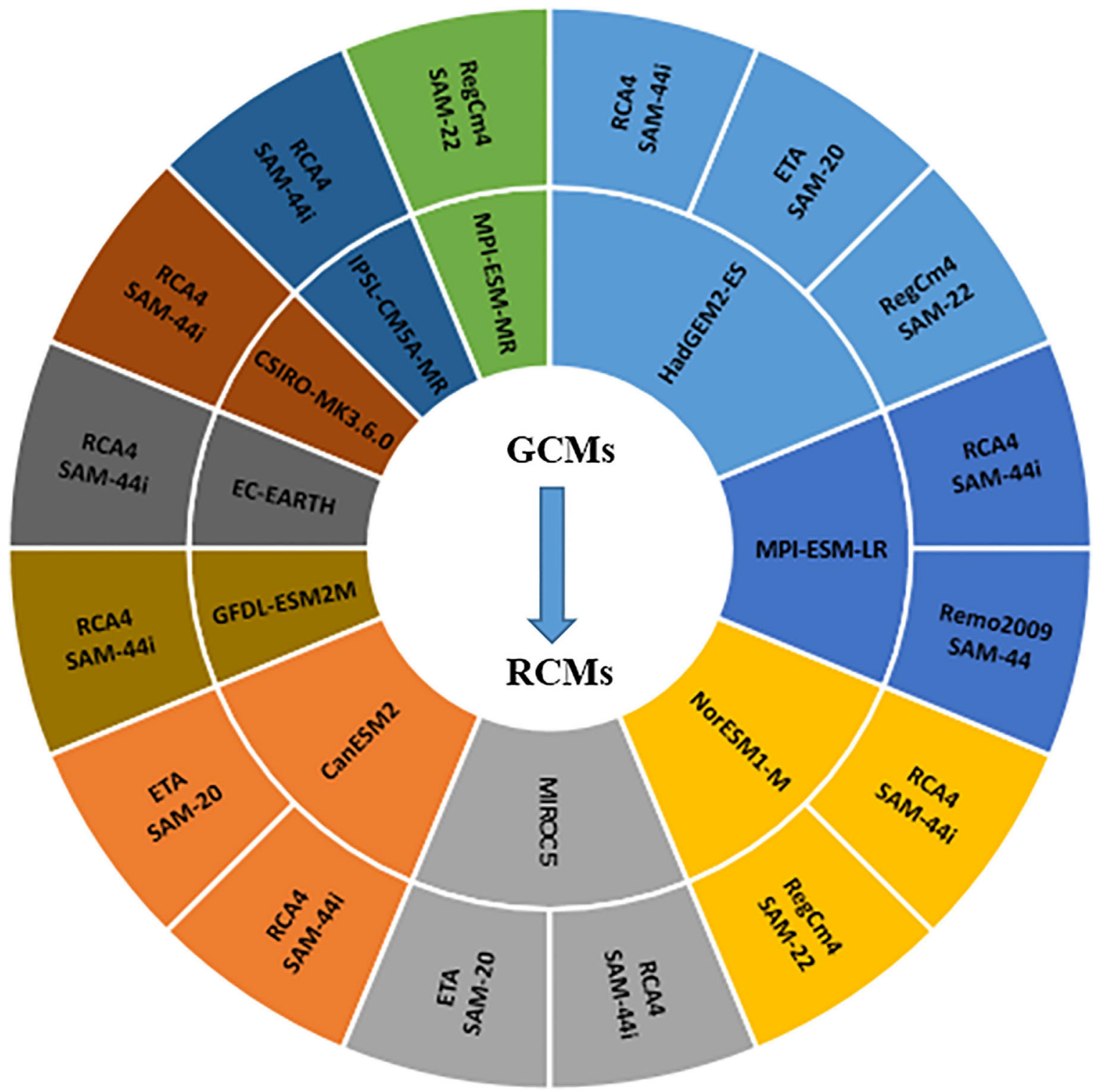

Figura 2 - Representação esquemática do resultado da busca. Para as condicionantes da pesquisa foram encontrados quatro RCMs aninhados com dez GCMs totalizando dezesseis combinações de modelos. O círculo mais externo representa os modelos regionais, seus domínios e os espaçamentos de grade. 
tion Pathways - RCP) conforme o último relatório do IPCC-AR5. A grande atratividade dos modelos regionais é a possibilidade de aumento da resolução. Enquanto os GCMs possuem uma resolução horizontal variando entre 250 a $600 \mathrm{~km}$, os RCMs variam de 10 a $50 \mathrm{~km}$ no máximo (IPCC, 2013; IPCC 2013a; Rockel, 2015).

As projeções regionalizadas são agrupadas por continentes ou partes de um continente denominadas domínios. Atualmente o globo terrestre é dividido em 14 domínios. No projeto Cordex podem ser encontrados dois sistemas de grades de modelos (regular e rotacionado). Esses sistemas são diferenciados pela letra "i" no final da descrição do domínio. Como por exemplo, o domínio da América do Sul SAM-44i (grade regular), ou apenas SAM-44 (grade rotacionada). O número 44 corresponde ao espaçamento da grade em graus $0,44^{\circ} \times 0,44^{\circ}(\mathrm{WCRP}$, 2019).

Isto posto, a presente pesquisa utilizou o Earth System Grid Federation (nó ESGF) para obter os arquivos das simulações climáticas. Trata-se de um sistema global de dados climáticos que permite acesso a dados de qualquer parte do mundo. A pesquisa procurou por simulações do domínio da América do Sul (SAM-20; SAM-22; SAM-44 e SAM-44i) que possuíssem dados de precipitação em frequência mensal da família do experimento Historical (ESGF, 2019). O resultado da busca está representado na Fig. 2.

A Tabela 1 apresenta informações complementares de cada modelo numérico envolvido nesta pesquisa. Para o presente estudo a combinação dos GCMs com os RCMs serão denominadas aqui simplesmente por "modelos". No entanto, deixa-se claro que os GCMs estabelecem apenas

Tabela 1 - Modelos climáticos disponíveis para a região estudada. A Tabela traz as informações do centro de pesquisa que desenvolve o modelo numérico, seu país de origem e a resolução espacial de cada modelo.

\begin{tabular}{|c|c|c|c|}
\hline Pais & Centro & Modelo & Resolução \\
\hline Canadá & $\mathrm{CCCma}$ & CanESM2 & $2.8^{\circ} \times 2.8^{\circ}$ \\
\hline França & IPSL & IPSL-CM5A-MR & $2.5^{\circ} \times 1.2^{\circ}$ \\
\hline Austrália & CSIRO & CSIRO-MK3.6.0 & $1.9^{\circ} \times 1.9^{\circ}$ \\
\hline Internacional & ICHEC & EC-EARTH & $1.1^{\circ} \times 1.1^{\circ}$ \\
\hline USA & NOAA-GFDL & GFDL-ESM2M & $2.5^{\circ} \times 2.0^{\circ}$ \\
\hline Japão & MIROC & MIROC5 & $1.4^{\circ} \times 1.4^{\circ}$ \\
\hline Alemanha & MPI-M & MPI-ESM-LR & $1.9^{\circ} \times 1.9^{\circ}$ \\
\hline Noruega & NCC, NMI & NorESM1-M & $2.5^{\circ} \times 1.9^{\circ}$ \\
\hline Reino Unido & $\mathrm{MOHC}$ & HadGEM2-ES & $1.9^{\circ} \times 1.2^{\circ}$ \\
\hline Noruega & NCC, NMI & NorESM1-M & $2.5^{\circ} \times 1.9^{\circ}$ \\
\hline Alemanha & MPI-M & MPI-ESM-MR & $1.9^{\circ} \times 1.9^{\circ}$ \\
\hline Suécia & SMHI & RCA4 V3 & $0.44^{\circ} \times 0.44^{\circ}$ \\
\hline Brasil & INPE & ETA V1 & $0.20^{\circ} \times 0.20^{\circ}$ \\
\hline Itália & ICTP & RegCM4-7 V0 & $0.22^{\circ} \times 0.22^{\circ}$ \\
\hline Alemanha & MPI-CSC & Remo2009 V1 & $0.44^{\circ} \times 0.44^{\circ}$ \\
\hline
\end{tabular}

Fonte: ESGF (2019). as condições de contorno dos RCMs, ou seja, os dados de saída dos GCMs são utilizados como condições iniciais para os RCMs (Freire et al., 2015).

Definido os modelos, o próximo passo consiste em aplicar o método de avaliação sazonal proposto por Silveira et al. (2019). As atividades do método estão descritas na Fig. 3.

As medidas estatísticas utilizadas na avaliação sazonal, cujas definições estão indicadas a seguir, conforme sugerido por Silveira et al. (2019), são a raiz do erro quadrático médio da contribuição percentual mensal em relação às chuvas anuais (RMSE_PC) e a correlação de Pearson (CORREL).

A raiz do erro quadrático médio percentual (RMSE_PC) é a raiz quadrada da média das diferenças individuais quadráticas, entre a contribuição percentual mensal das chuvas modeladas e observadas para os seus totais anuais, sendo definida pela Eq. (1):

$$
\operatorname{RMSE}_{P} C=\sqrt{\frac{1}{n} \sum_{i=1}^{n}\left(\frac{100 \cdot P_{i}}{\sum_{i=1}^{n} P_{i}}-\frac{100 \cdot A_{i}}{\sum_{i=1}^{n} A_{i}}\right)^{2}}
$$

onde $n$ são os meses, $P$ é previsão de cada mês e $A$, a observação. Valores grandes de RMSE_PC representam erros maiores nos campos previstos e valores próximos de zero indicam uma previsão quase perfeita. Elevando ao quadrado o termo da diferença, o RMSE_PC tende a dar maior peso às grandes discrepâncias entre os campos observados e previstos (Silveira et al., 2019).

A correlação pode assumir valores entre -1 e 1 que indica, respectivamente, perfeita anticorrelação e perfeita correlação, com a ausência total de correlação correspondendo a um valor nulo. Este índice tem a capacidade de detectar correspondência de fase entre as séries, sendo, por construção, insensível a erros de viés. É dado conforme a Eq. (2):

$$
\text { CORREL }=\frac{\sum_{i=1}^{n}\left(A_{i}-\bar{A}\right)\left(P_{i}-\bar{P}\right)}{\sqrt{\sum_{i=1}^{n}\left(P_{i}-\bar{P}\right)^{2} \sum_{i=1}^{n}\left(A_{i}-\bar{A}\right) 2}}
$$

Após o cálculo desses dois índices foi calculado um índice de avaliação ponderada para que os modelos possam ser classificados, tal que:

$$
\begin{array}{r}
A V A L_{s}=\propto_{c} \cdot\left(\frac{C O R R E L-C O R R E L_{M I N}}{\text { CORREL }_{M A X}-C O R R E L_{M I N}}\right) \\
+\propto_{r} \cdot\left(\frac{R M S E_{-} P C_{M A X}-R M S E_{-} P C}{R M S E_{-} P C_{M A X}-R M S E_{M I N}}\right) \\
\propto_{c}+\propto_{r}=1
\end{array}
$$

Sendo, CORRELMIN a menor correlação obtida entre os modelos e CORRELMAX a máxima correlação, assim 


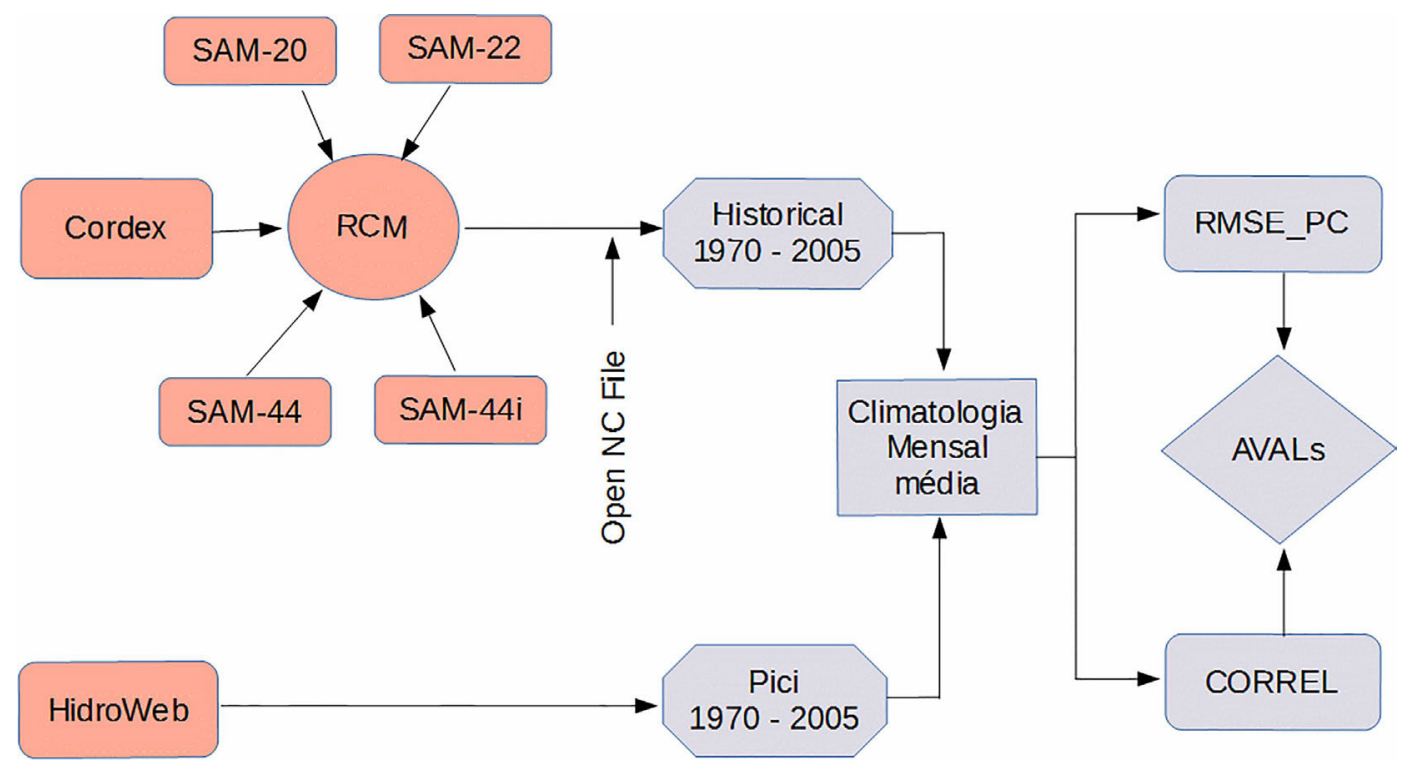

Figura 3 - Fluxograma da aplicação do método de avaliação. A pesquisa é fundamentada em dois eixos, um relacionado a dados observados (HidroWeb) e outro relacionado a dados simulados (Cordex). No nó ESGF os dados são fornecidos em arquivos digitais no formato NetCDF e foi utilizado o Software Open NC File (Agrimetsoft, 2018) para extrair as informações em formato.xlxs para as coordenadas da estação Pici (latitude: -3.75 ; longitude: -38.58 ). A parti disso, foi possível estabelecer as relações entre a precipitação simulada de cada modelo para o clima presente e o observado. Utilizou-se o período Historical (1970 a 2005 - 35 anos) para realizar as comparações. Adaptado de Silveira et al. (2019).

como RMSE_PCMAX é o máximo erro quadrático médio percentual dos modelos e RMSE_PCMIN, o mínimo (Silveira et al., 2019).

As variáveis $\alpha_{c}$ e $\alpha_{r}$ assumem valores entre 0 e 1 (conforme Eq. (4)), para $\alpha_{c}>\alpha_{r}$, a CORREL exerce maior influência na avaliação do modelo, enquanto para $\alpha_{r}>\alpha_{c}$, o RMSE_PC dos modelos possui maior peso na avaliação. Já se $\alpha_{r}=\alpha_{c}=0,5$ as duas métricas utilizadas neste trabalho exercem o mesmo efeito sobre o valor de AVALs. Nos casos extremos, ao se considerar $\alpha_{r}=0$ então $\alpha_{c}=1$, apenas a CORREL foi usada na avaliação dos modelos, assim como, se $\alpha_{r}=1$ então $\alpha_{c}=0$, apenas o RMSE_PC foi usado na avaliação dos modelos. Neste estudo será aplicado o valor de 0,5 para cada variável.

\section{Resultados e Discussão}

A Tabela 2 apresenta os resultados encontrados e classifica os modelos segundo o índice AVALs. O modelo regional ETA apresentou os maiores valores de AVALs sugerindo que este modelo possui a melhor representação dos padrões de variação sazonal da precipitação para o município estudado. Dentro todos os modelos, O ETA aninhado com o CanESM2 liderou a avaliação sazonal proposta por Silveira et al. (2019). Esse fato pode ser justificado por conta da resolução utilizada pelo modelo que é de $0,20^{\circ} \times 0,20^{\circ}$, ou aproximadamente $22,5 \mathrm{~km}$ por $22,5 \mathrm{~km}$. De todas as resoluções encontradas nas simulações do Projeto Cordex para o domínio da América do Sul essa é a mais refinada.
Como forma didática de realizar a discussão dos resultados, a Fig. 4 apresenta uma divisão gráfica do grau de representatividade para o clima presente. A partir daqui a discussão dos resultados será realizado por grupo de boa, regular e baixa representatividade dos modelos.

Tabela 2 - Classificação de representatividade segundo índice AVALs. Constata-se que o valor do AVALs é proveniente da combinação do CORREL e RMSE_PC. Quanto maior o CORREL e menor o RMSE_PC, maior o valor de AVALs.

\begin{tabular}{lccc}
\hline Modelos & AVALs & CORREL & RMSE_PC \\
\hline CANESM2_ETA & 1 & 0.96 & 3.01 \\
HADGEM2-ES_ETA & 0.98 & 0.94 & 3.01 \\
HADGEM2-ES_RegCM4 & 0.97 & 0.96 & 3.26 \\
MIROC5_ETA & 0.88 & 0.92 & 3.76 \\
HADGEM2-ES_RCA4 & 0.8 & 0.86 & 3.86 \\
GFDL-ESM2M_RCA4 & 0.56 & 0.77 & 5.1 \\
CSIRO-MK3-6-0_RCA4 & 0.55 & 0.78 & 5.29 \\
CM5A-MR_RCA4 & 0.5 & 0.77 & 5.58 \\
MIROC5_RCA4 & 0.5 & 0.77 & 5.62 \\
NORESM1-M_RegCM4 & 0.5 & 0.8 & 5.87 \\
NOrESM1-M_RCA4 & 0.39 & 0.7 & 5.89 \\
CANESM2_RCA4 & 0.39 & 0.69 & 5.83 \\
MPI-ESM-MR_RegCM4 & 0.24 & 0.67 & 6.94 \\
EC-EARTH_RCA4 & 0.19 & 0.58 & 6.5 \\
MPI-ESM-LR_REMO2009 & 0.12 & 0.6 & 7.3 \\
MPI-ESM-LR_RCA4 & 0 & 0.51 & 7.49 \\
\hline
\end{tabular}




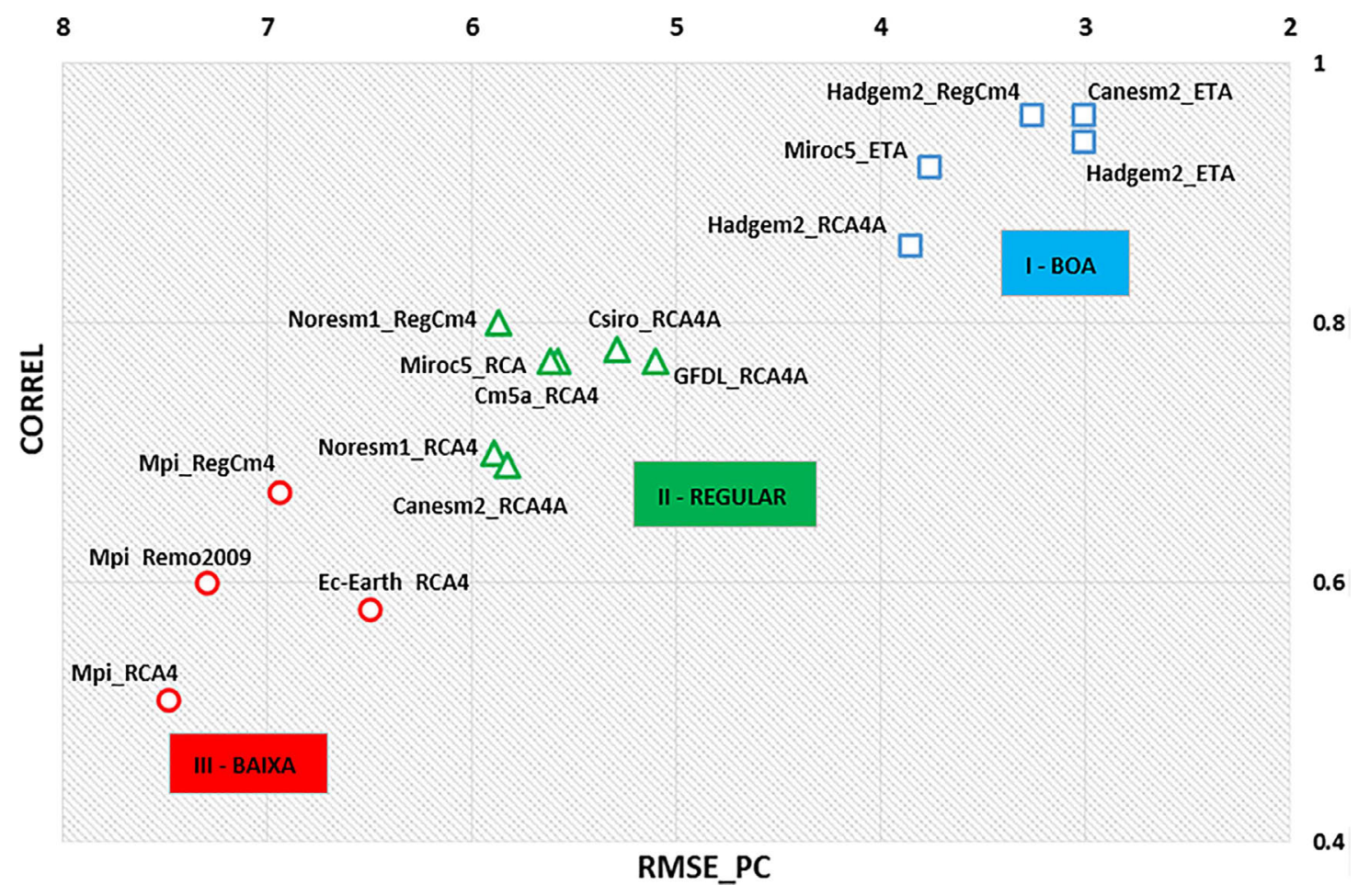

Figura 4 - Classificação segundo grau de representatividade. Os modelos de boa representatividade correspondem aos da região azul, verde para os regulares, e vermelho para os modelos com baixa representatividade do clima presente. Os modelos com boa representatividade possuem CORREL maiores que 0,8 e RMSE_PC abaixo de 4\%. Para os modelos com representatividade regular a CORREL está compreendida entre 0,6 e 0,8 com RMSE_PC abaixo de 6\%. Já os modelos que apresentaram baixo desempenho se concentraram nas áreas de baixa CORREL e alto RMSE_PC.

Na Fig. 4 destaca-se ainda a importância das condições de contorno dos RCMs. O bom desempenho do CanESM2 na representatividade do clima presente já foi constatado por alguns autores: Silveira et al. (2013) aplicaram esse mesmo método de avaliação para os modelos do CMIP5 e avaliaram o CanESM2 como o melhor modelo para representar a região Nordeste do Brasil (NEB); Guimarães et al. (2016) analisaram o desempenho das projeções do CMIP5 e do Cordex para o Nordeste Brasileiro e também chegaram à conclusão que o CanESM2 é um dos modelos mais apropriados. Apesar do modelo Canadense liderar a classificação no presente estudo, é possível notar a predominância do HadGEM2-ES em aninhamento de modelos com grau de boa representatividade. Entretanto, em estudo sobre mudança climática na bacia do Rio São Francisco, Silveira et al. (2016) informa que o modelo HadGEM2-ES apresentou AVALs bem aquém indicando que o mesmo não representava adequadamente a sazonalidade da precipitação naquela bacia. Corroborando ainda a importância da condição de contorno, na outra vertente, tem-se a predominância dos modelos MPI-ESM no aninhamento de modelos de baixa representatividade.

Ampliando a análise da comparação, apresenta-se o comportamento das climatologias dos modelos para escala sazonal. Para melhor a visualização preferiu-se dividir a exibição por graus de representatividade. A Fig. 5 apre- senta os modelos com boa representatividade, a Fig. 6 os com regular representatividade e a Fig. 7 os modelos com baixa representatividade.

$\mathrm{Na}$ Fig. 5, pode-se constatar que a maioria dos modelos possuem uma boa aderência a climatologia observada, com exceção do modelo HadGEM2-ES_RegCM4. No primeiro semestre do ano o modelo superestima a precipitação, contudo conserva a semelhança da curva com a curva observada. Isso é possível porque a formulação matemática da CORREL e RMSE_PC permitem. Considerando de que se trata de comparações de proporções, e que nesse caso especifico as proporções se mantiveram, o método inicialmente não apontou esse viés úmido. Por isso, o presente estudo faz o alerta da importância do processo de remoção do viés antes da aplicação em pesquisa que buscam avaliar impactos das mudanças climáticas. O interessante é que as simulações do clima futuro passem por essas correções antes de sua utilização.

Na Fig. 6 já é possível notar um maior espalhamento das climatologias. $\mathrm{O}$ único período que existe consenso dos valores corresponde os meses de julho a outubro. As maiores distorções ficam por conta dos meses da pré-estação chuvosa (dezembro a fevereiro).

Na Fig. 7 pode-se constatar a falta de aderência a climatologia observada. Da mesma forma dos modelos de representatividade regular, os modelos com baixa representatividade possuem unanimidade entre os meses julho a 


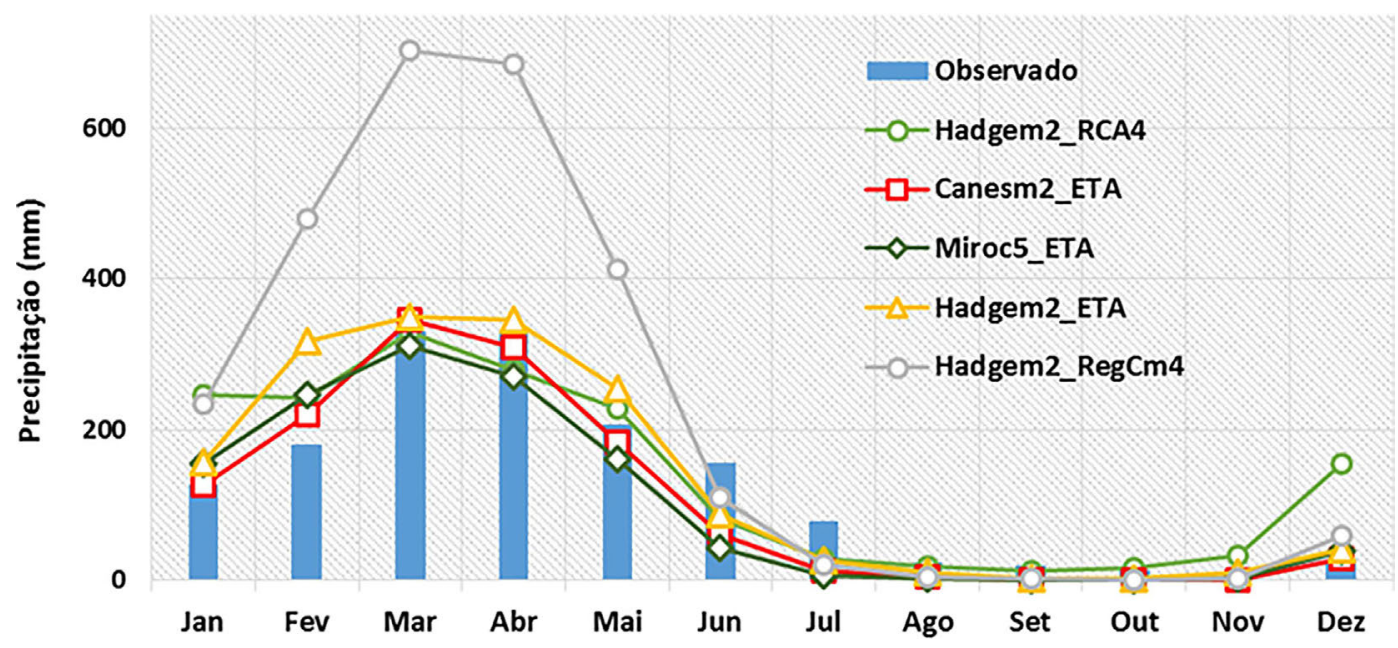

Figura 5 - Sazonalidade dos modelos com boa representatividade (1970 a 2005). Os dados em barra correspondem a dados observados (HidroWeb) e os dados em linhas correspondem ao modelos climáticos (Cordex).

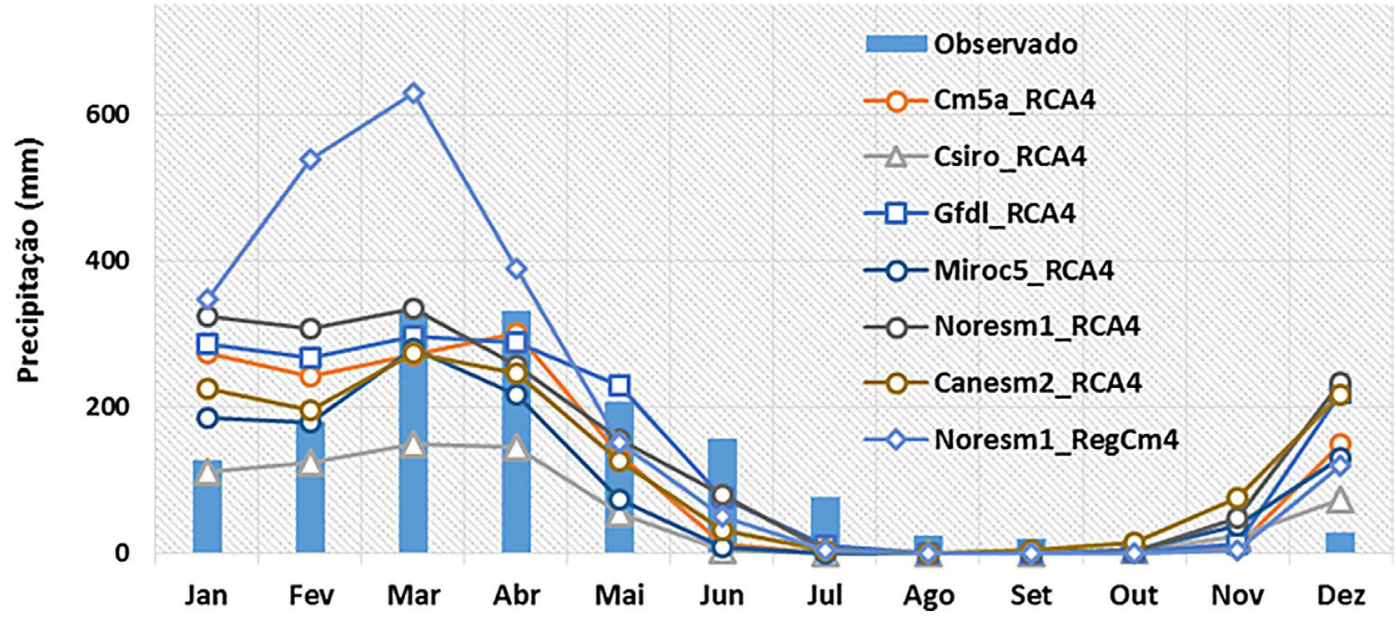

Figura 6 - Sazonalidade dos modelos com regular representatividade (1970 a 2005). Os dados em barra correspondem a dados observados (HidroWeb) e os dados em linhas correspondem ao modelos climáticos (Cordex).

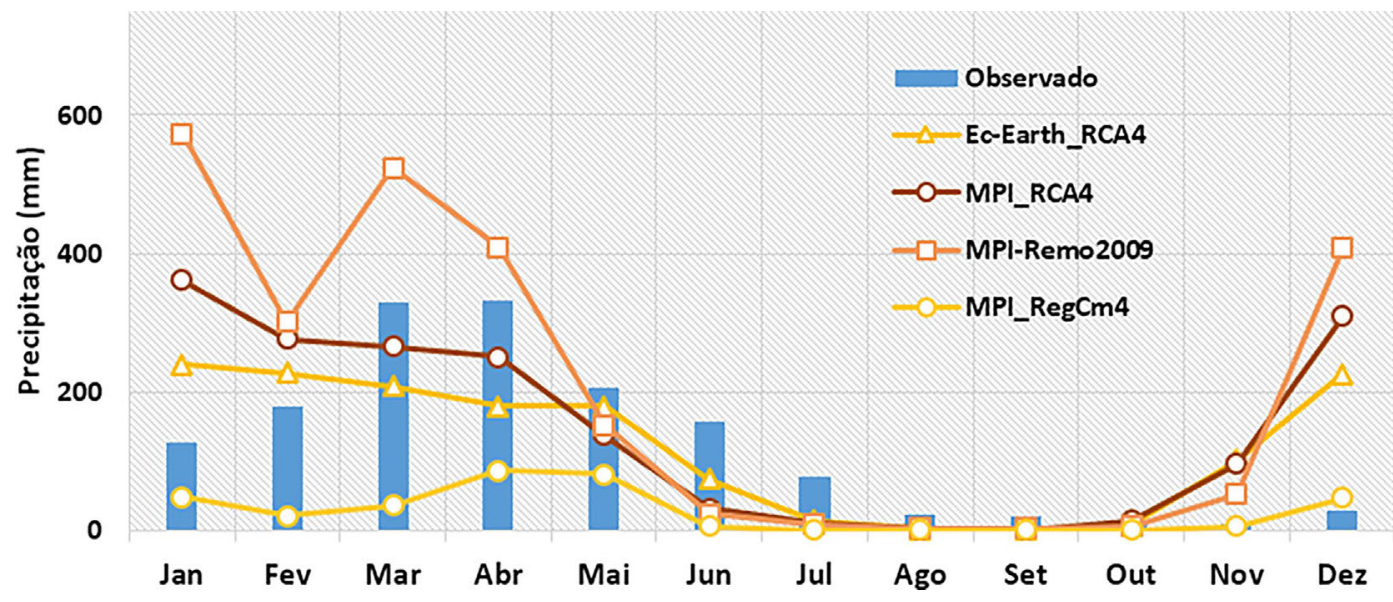

Figura 7 - Sazonalidade dos modelos com baixa representatividade (1970 a 2005). Os dados em barra correspondem a dados observados (HidroWeb) e os dados em linhas correspondem ao modelos climáticos (Cordex). 
outubro. A tradução desse fato é que os modelos entendem que esses meses a ocorrência de precipitação é escassa. Contudo a maioria dos modelos (regular e baixa representatividade) superestimam os valores de precipitação com a chegada da pré-estação chuvosa e para a estação chuvosa existe um nível de ruído elevado na informação. Com isso, o reflexo dessas características é o baixo desempenho dos modelos.

\section{Considerações Finais}

O modelo regional ETA apresentou o melhor desempenho na representatividade do clima presente para o município estudado. A condução pelo modelo global CanESM2, segundo o índice AVALs proposto por Silveira et al. (2019), se apresentou como melhor simulação para o período avaliado com um CORREL de 0,96 e RMSE_PC de 3,01\% corroborando com o encontrado por Silveira et al. (2013) para região Nordeste do Brasil.

O presente trabalho identificou a importância do papel dos GCMs nas projeções do RCMs (condições de contorno). Foi constatado na Figura 5 que dos cinco modelos que apresentaram uma boa representatividade o HadGEM2-ES fornece as condições de contorno para três deles, enquanto para os modelos de baixa representatividade (Fig. 7) o MPI-ESM-LR/MR demonstrou predominância na condução. Isso leva a deduzir que para obtenção de uma boa projeção regionalizada, os dados de contorno também devem possuir boa aderência a climatologia estudada.

Outro aspecto relevante encontrado foi a resolução dos modelos regionais. Ficou evidenciado que a classificação está relacionada diretamente com o espaçamento das grades, quanto melhor posicionado na classificação maior a resolução do modelo.

Por fim, a recomendação do presente trabalho é que sejam adotados modelos com boa e regular representatividade em pesquisas desenvolvidas para avaliar impacto das mudanças climáticas no município estudado.

\section{Referências}

AGRIMETSOFT, Agricultural and Meteorological Software. Open NC File Software [Computer software]. 2018. Disponível em: https://agrimetsoft.com/open_nc_file_for_ coordinates.aspx.

ANA - Agência Nacional De Águas. HidroWeb: Sistemas de Informações Hidrológicas. 2019. Disponível em: http:// www.snirh.gov.br/hidroweb/apresentacao. Acesso em 31 maio 2019.

BENESTAD, R. Downscaling Climate Information.” Oxford Research Encyclopedia of Climate Science, Oxford University Press, Oxford Research Encyclopedia of Climate Science, July. 2016. https://doi.org/10.1093/acrefore/ 9780190228620.013.27.
CEARÁ. Instituto de Pesquisa e Estratégia Econômica do Ceará (IPECE). Perfil Municipal 2017 Fortaleza, 2018, Fortaleza. Disponível em: https://www.ipece.ce.gov.br/wp-con tent/uploads/sites/45/2018/09/Fortaleza_2017.pdf. Acesso em 23 março de 2019.

COSTA, L.C. Dimensionamento de Reservatórios para Sistemas de Abastecimento de Água Pluvial para a Região dos Campos Gerais Tendo em Vista Cenários de Mudanças Climáticas. Dissertação de Mestrado em Saneamento Ambiental e Recursos Hídricos, Universidade Estadual de Ponta Grossa, Ponta Grossa, 2016. 76 p. Disponível em: http://tede2.uepg.br/jspui/handle/prefix/28. Acesso em 23 agosto de 2019.

ESGF - Earth System Grid Federation. National Supercomputer Centre at Linköping University (NSC), 2019. Disponível: https://esg-dn1.nsc.liu.se/search/esgf-liu/. Acesso em 17 de julho de 2019.

FORTALEZA. Drenagem e Manejo de Águas Pluviais Urbanas do Município de Fortaleza. Fortaleza: Secretaria Municipal de Urbanismo e Meio Ambiente, 2015. Disponível em: https://urbanismoemeioambiente.fortaleza.ce. gov.br/images/urbanismo-e-meio-ambiente/infocidade/pla no_municipal_de_drenagem.pdf. Acesso em 23 agosto de 2019.

FREIRE, J.L.M.; FREITAS, S.R.; COELHO, C.A.S. Calibração do modelo regional BRAMS para a previsão de eventos climáticos extremos. Revista Brasileira de Meteorologia, v. 30, n. 2, p. 158-170, 2015.

GUIMARAES, S.O.; COSTA, A.A.; VASCONCELOS JÚNIOR, F.C.; SILVA, E.M.; SALES, D.C.; ARAÚJO JÚNIOR, L. M.; SOUZA, S. G. Projeções de mudanças climáticas sobre o nordeste brasileiro dos modelos do CMIP5 e do CORDEX. Revista Brasileira de Meteorologia, v. 31, n. 3, p. 337-365, 2016.

IPCC - Intergovernmental Panel on Climate Change. Climate Change 2001: The Scientific Basis. Contribution of Working Group I to the Third Assessment Report of the Intergovernmental Panel on Climate Change. Cambridge: Cambridge University Press, 2001.

IPCC - Intergovernmental Panel on Climate Change. General Guidelines on the Use of Scenario Data for Climate Impact and Adaptation Assessment. Task Group on Data and Scenario Support for Impact and Climate Assessement (TGICA), 66 p, 2007. Disponível em: http://www.ipccdata.org/guidelines/TGICA_guidance_sdciaa_v2_final.pdf. Acesso em 26 março de 2019.

IPCC - Intergovernmental Panel on Climate Change. Climate change 2013: The Physical Science Basis. Contribution of Working Group I to the Fifth Assessment Report of the Intergovernmental Panel on Climate Change. STOCKER, T.F., et al. (eds). Cambridge: Cambridge University Press, 1535 pp., 2013.

IPCC - Intergovernmental Panel on Climate Change. What is a GCM? Data Distribution Centre, 2013a. Disponível em: https://www.ipcc-data.org/guidelines/pages/gcm_guide. html. Acesso em 17 de maio de 2020.

PBMC - Painel Brasileiro de Mudanças Climáticas. Mudanças Climáticas e Cidades. Relatório Especial do Painel Brasileiro de Mudanças Climáticas. RIBEIRO, S.K.; SANTOS, A.S. (Eds). COPPE, Rio de Janeiro, 116 p., 2016. 
RAMOS, A.M. Influência das Mudanças Climáticas Devido ao Efeito Estufa na Drenagem Urbana de uma Grande Cidade. Tese de Doutorado, Programa de Pós-Graduação em Engenharia Civil, Universidade Federal de Pernambuco, Recife, 2010. Disponível em: https://repositorio.ufpe. br/handle/123456789/5111. Acesso em 23 março de 2019.

ROCKEL, B. The Regional Downscaling Approach: a Brief History and Recent Advances. Current Climate Change Reports 1, 22-29, 2015. https://doi.org/10.1007/s40641-0140001-3.

ROSSI, F. A.; DUMKE, E.; KRUGER, E. L. Atualização do ano climático de referência para Curitiba. In: $\mathbf{X}$ Encontro Nacional e VII Encontro Latino Americano de Conforto no Ambiente Construído. Natal, 2009. Disponível em: https://www.researchgate.net/profile/Eduardo_Krueger/pub lication/268354042_ATUALIZACAO_DO_ANO_CLIMA TICO_DE_REFERENCIA_PARA_CURITIBA/links/ 5513ef7f0cf23203199ccb70/ATUALIZACAO-DO-ANOCLIMATICO-DE-REFERENCIA-PARA-CURITIBA.pdf. Acesso em 23 março de 2019.

ROSENZWEIG C.; SOLECKI, W.; ROMERO-LANKAO, P.; MEHROTRA, S.; DHAKAL, S.; BOWMAN, T.; ALI IBRAHIM, S. ARC3.2 Summary for City Leaders. Urban Climate Change Research Network. Columbia University. New York, 2015. Disponível em: https://unfccc. int/files/parties_observers/submissions_from_observers/ application/pdf/787.pdf. Acesso em 02 de abril de 2020.
SILVEIRA, C.S.; SOUZA FILHO, F.A.; COSTA, A.A.; CABRAL, S.L. Avaliação de desempenho dos modelos do CMIP5 quanto à representação dos padrões de variação da precipitação no século XX sobre a região Nordeste do Brasil, Amazônia e bacia do Prata e análise das projeções para o cenário RCP8.5. Revista Brasileira de Meteorologia, v. 28 , n. 3, p. 317-330, 2013.

SILVEIRA, C.S.; SOUZA FILHO, F.A.; MARTINS, E.S.P.R.; OLIVEIRA, J.L.; COSTA, A.C.; NOBREGA, M.T.; SOUZA, S.A.; SILVA, R.B.F.V. Mudanças climáticas na bacia do rio São Francisco: Uma análise para precipitação e temperatura. Revista Brasileira de Recursos Hídricos, v. 21, n. 2, p. 415-428, 2016.

SILVEIRA, C.S.; VASCONCELOS JÚNIOR, F.C.; SOUZA FILHO, F.A.; GUIMARAES, S.O.; MARCOS JÚNIOR, A.D.; REIS, G.N.L.; PORTO, V.C. Performance evaluation of AR5-CMIP5 models for the representation of seasonal and multi-annual variability of precipitation in Brazilian hydropower sector basins under RCP8.5 scenario, Hydrological Sciences Journal, 64:11,1279-1296, 2019.

WCRP - World Climate Research Programme. Coordinated Regional Climate Downscaling Experimente - Cordex, 2019. Disponível em: https://cordex.org/. Acesso em 12 de junho de 2019.

License information: This is an open-access article distributed under the terms of the Creative Commons Attribution License (type CC-BY), which permits unrestricted use, distribution and reproduction in any medium, provided the original article is properly cited. 